\title{
A complex oncosurgical approach to increasing the resectability of colorectal cancer metastases - a case report
}

\author{
Vladislav Treska ${ }^{\mathrm{a}}$, Jarmil Safranek ${ }^{\mathrm{a}}$, Daniel Lysak ${ }^{\mathrm{b}}$, Hynek Mirka ${ }^{\mathrm{c}}$, Tomas Skalicky ${ }^{\mathrm{a}}$,Frantisek Slaufc, Ondrej Hes ${ }^{\mathrm{d}}$
}

\begin{abstract}
Aim. In this case report, the authors aim to demonstrate the success of recent methods in the radical treatment of a patient with primary inoperable liver and subsequent colorectal cancer pulmonary metastases.

Methods. A 75 year old patient with inoperable bulky metastasis in the right hepatic lobe and insufficient future remnant liver volume was indicated for a stage procedure in the liver parenchyma. Embolization of the right branch of the portal vein was first performed with subsequent administration of stem cells into the contralateral liver lobe. Following compensatory growth of the left liver lobe, right-sided hepatectomy was performed with subsequent adjuvant oncological treatment. Six months after the surgery, a metastasis developed in the right pulmonary lobe which was solved by metastasectomy.

Results. The patient, one year after the diagnosis of inoperable liver metastasis, is completely healthy and free of signs of disease recurrence.

Conclusion. A comprehensive oncosurgical approach using up-to-date diagnostic and treatment options may offer patients with metastatic colorectal cancer, radical treatment with the hope of long-term quality survival.
\end{abstract}

Key words: colorectal cancer liver metastases, portal vein embolization, stem cells administration, pulmonary metastasectomy

Received: August 9, 2011; Accepted with revision: January 3, 2012; Available online: January 31, 2012

http://dx.doi.org/10.5507/bp.2012.005

${ }^{a}$ Department of Surgery, University Hospital and Medical Faculty in Pilsen, Czech Republic

${ }^{b}$ Department of Haematooncology, University Hospital and Medical Faculty in Pilsen

'Department of Radiology, University Hospital and Medical Faculty in Pilsen

${ }^{d}$ Department of Pathology, University Hospital and Medical Faculty in Pilsen

Corresponding author: Vladislav Treska, e-mail: treska@fnplzen.cz

\section{INTRODUCTION}

Surgical treatment of colorectal cancer liver metastases (CLMs) is a gold standard offering patients quality long-term survival. From our own experience and the literature, a five-year survival in radically operated patients ( $\mathrm{R} 0$ resection) is achieved in $35-60 \%$ of cases $^{1-3}$. Nevertheless, a large percentage (up to $80 \%$ ) of patients diagnosed with CLMs are primarily inoperable due to the extent and location of metastases in the liver and body. In some patients with primarily surgically inoperable cancer whose life expectancy is very short, we can significantly extend their quality of life by means of procedures that result in the final radical surgery.

\section{MATERIAL AND METHOD}

A 75 year old patient J.K. was referred to our clinic from another department in June 2010 to evaluate the findings in his liver. This patient was found to have a metastasis in the right liver lobe during the check-up using ultrasound examination (USG). The patient had undergone resection of the colon in May 2008 with subsequent oncological therapy. He was in general good health with the exception of treatment for coronary artery disease with unstable angina pectoris syndrome and mild mitral insufficiency. He also had hypertension. A cerebrovascular accident was found in the medical history too, but currently he was free of any neurological symptomatology and the ultrasound finding in the internal carotid arteries was normal. A bulky metastasis $8 \mathrm{~cm}$ in size was found during the performed high-resolution computerized tomography (HRCT) of the abdomen (Fig. 1) in the right liver lobe. The volume of the liver was $1777 \mathrm{~mL}$, and the volume of the left liver lobe was less than $30 \%$ of the total liver volume (Somatom Definition, Siemens, Germany). Due to the borderline future remnant liver volume of the liver parenchyma (FRLV) we decided to perform a phase procedure which consisted of embolization of the right branch of the portal vein (PVE), followed by subsequent leukapheresis, application of hematopoietic stem cells (HSC) and right hepatectomy. The patient was instructed in detail about the entire procedure. He agreed and signed the informed consent.

We performed the PVE by the transparietal approach using Histoacryl (Braun, Germany) mixture: Lipiodol (Cedex, France) in a ratio of 1:10 (Fig. 2). Peripheral stem haematopoietic cells (HSC) collected using the apheresis of the peripheral blood were the source of 


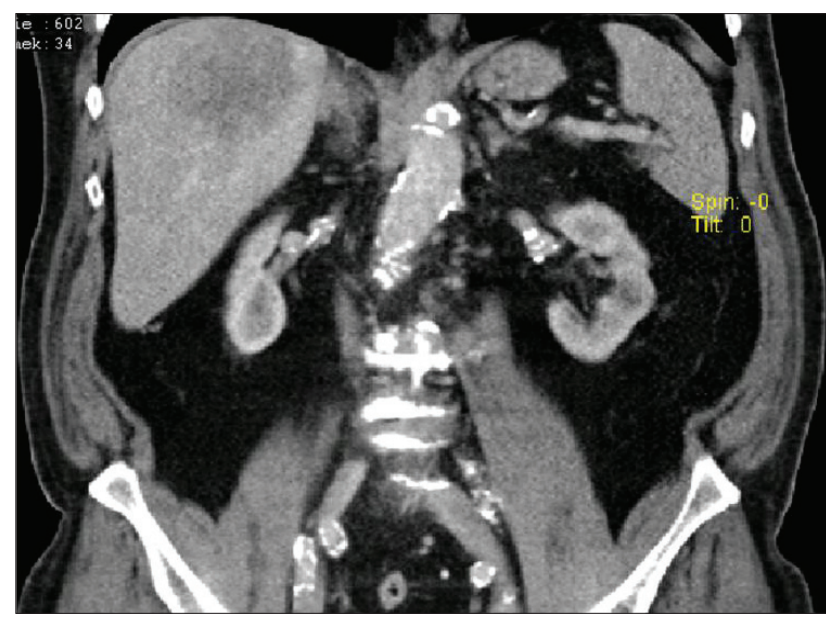

Fig. 1. Hepatic HRCT with a bulky metastasis of the right liver lobe.

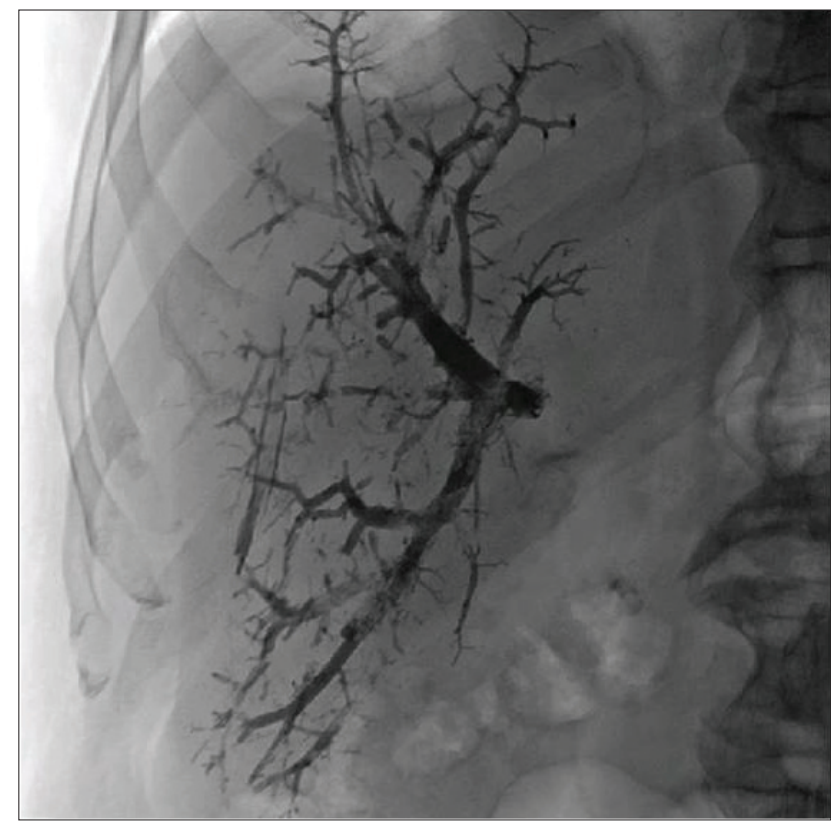

Fig. 2. PVE of the right branch of the portal vein.

HSC. The patient was stimulated prior to sampling with granulopoiesis growth factor (G-CSF, Neupogen, Amgen Europe BV, Breda, The Netherlands) in a dose of $10 \mathrm{mg} /$ $\mathrm{kg}$ /day s.c. administered as a single daily dose for 4 days. From the fourth day after the Neupogen administration circulating stem cells (detected by flow cytometry as CD34+ cells) were monitored in circulating peripheral blood. On the fifth day after the administration of Neupogen, one high-volume leukapheresis in the continual blood elements separator Cobe Spectra (CaridianBCT, Lakewood, CO, USA) was performed using a dialyzation catheter inserted into the vena femoralis, a program for collection of mononuclear leukocytes (MNC program, software version 6.1). Approximately three volumes of the patient's blood were processed, citric acid based solution and citric acid was used as an anticoagulant agent (ACD-A, Baxter, Deerfield, IL, USA) in the ratio of 1:12

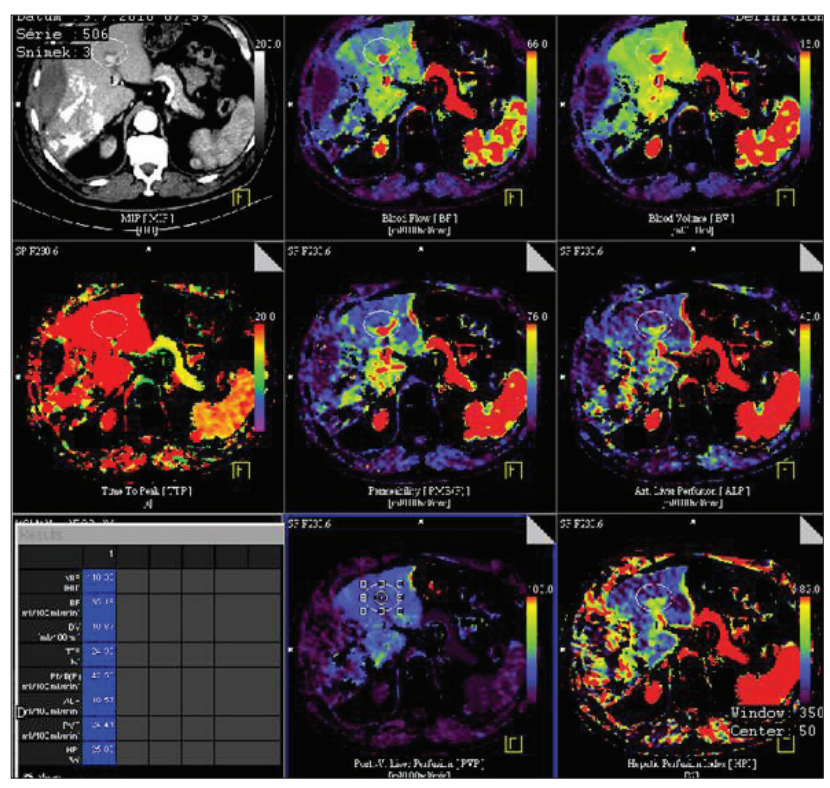

Fig. 3. Perfusion CT 14 days after the PVE with administration of HSC - FRLV $=45 \%$ of the overall liver volume, small subcapsular hematoma of the right liver lobe.

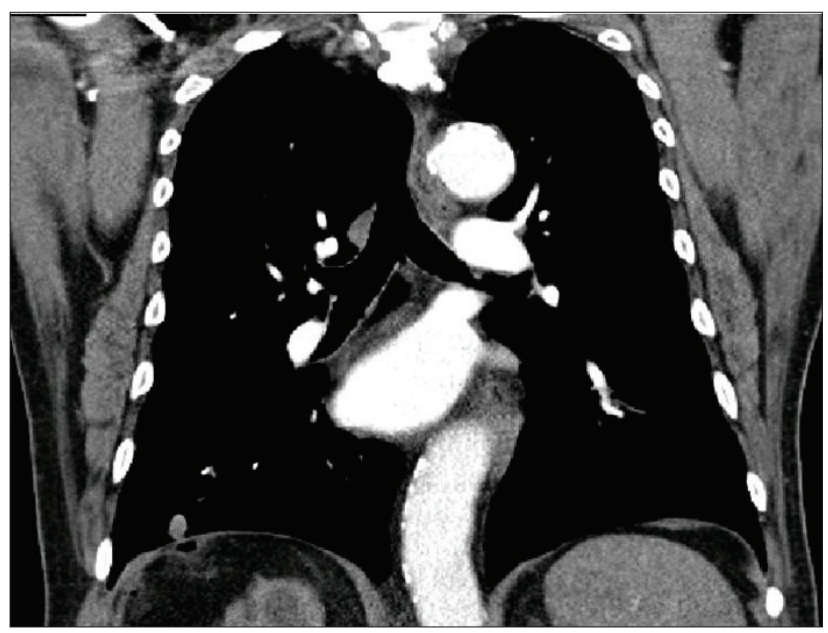

Fig. 4. HRCT of the lung with the evidence of metastasis in the right lower pulmonary lobe.

to $1: 14$ to whole blood. To prevent citrate toxicity calcium was supplemented into the backward line (fractionated, total dose 10 to $20 \mathrm{~mL}$ of $\mathrm{CaCl} 2$ ). The obtained product was analyzed in the laboratory and basic quality parameters were determined: volume, concentration and absolute leukocyte count and CD34+ cells, erythrocyte and thrombocyte count, viability of CD34+ cells, sterility, and CD133+ cell count. A collection of samples for the determination of all tests was performed within the closed system. Prior to mobilization of HSCs all patients had undergone an examination to exclude blood transmissible diseases (HIV, HBV, HCV, syphilis). No handling with the product was further performed and it was stored till the next day at 2 to $8{ }^{\circ} \mathrm{C}$ under continual monitoring of storage conditions. On the next day we administered under general anesthesia in the operating room $114 \mathrm{~mL}$ of the product with $52.3 \times 10^{6}$ of CD $133+$ and $66.3 \times 10^{6}$ 
CD34+ HSC into the left non-embolized branch of the portal vein via the ileocolic vein.

We monitored the growth of the contralateral liver lobe using the HRCT volumometry of the liver at weekly intervals following the PVE and administration of HSC. A rise in FRLV to $45 \%$ of the total liver volume occurred 14 days after the PVE and the administration of HSC (Fig. 3) and we performed liver surgery. In the right liver lobe a bulky metastasis was found, $8 \mathrm{~cm}$ in diameter that had infiltrated the right liver vein. The left liver lobe was sufficiently increased and intraoperative ultrasound detected no additional metastases in the liver parenchyma. We performed a right-sided hepatectomy. Histologically, a metastasis of well- differentiated colorectal adenocarcinoma was identified. The postoperative course was uneventful and the patient was discharged on the $10^{\text {th }}$ postoperative day for home care. During the check-up one month after the surgery, the patient was in a generally good condition, the hepatic HRCT found no metastases and tumor markers were normal. The patient was monitored and treated at the regional oncological department using the FOLFOX regime. During the check-up at our clinic in March 2011 a suspect solitary metastasis of $1.3 \mathrm{~cm}$ in diameter was found in the lower pulmonary lobe in the right during the HRCT but, the hepatic parenchyma was free of metastases (Fig. 4). We decided to perform metastasectomy of the pulmonary focus and this was carried out in April 2011 from the anterolateral thoracotomy with removal of the regional lymphatic nodes. Histological examination verified a metastasis of colorectal adenocarcinoma without involvement of the lymphatic nodes.

\section{RESULTS}

The patient was discharged for home care on the 8th day following the surgery in a generally good condition. Currently, the patient is under the care of the regional oncological department without adjuvant oncological therapy. The last check-up at our clinic was in July 2011, when the patient had no problems and his clinical status was very good. Hepatic, pulmonary and mediastinal HRCT was free of signs of the underlying disease recurrence.

\section{DISCUSSION}

Just a few years ago a finding of a little bulky CLM with a small FRLV or dissemination of the tumor with involvement of other organs would be considered insolvable due to the small hepatic reserve or generalization of the underlying disease. A radical oncosurgical approach has significantly changed the attitude towards these patients and provided them with prolongation of their quality of life $^{4,5}$. The Czech Republic is unfortunately the leading country in incidences of colorectal cancer. We can expect, while presuming generally the occurrence of synchronous and metachronous colorectal cancer liver metastases in $60-70 \%$ of these patients and a significant incidence of colorectal cancer liver metastases in our population as well. Unfortunately, still only $15-25 \%$ of patients can primarily undergo surgery at the time when a CLM is diagnosed. The causes of inoperability in patients include on the one hand the volume of the CLM with insufficient FRLV, and on the other hand the involvement of other organs due to the metastatic process. An insufficient FRLV is generally considered as the volume of healthy liver of less than $30 \%$. The larger FRLV (40-60\%) is required in the liver affected by e.g. chemotherapy / cirrhosis depending on the degree of the liver disease $e^{6,7}$. In patients with insufficient FRLV, the PVE is indicated as the first step before a large liver resection. Usually hypertrophy of liver parenchyma develops following the PVE at intervals of 2 to 8 weeks by $20-46 \%$, and $70-100 \%$ of patients can undergo liver resection in the interval of 4 to 6 weeks after the PVE (ref. ${ }^{8,9}$ ). The long-term results of liver resections after the PVE are practically comparable with the primary surgeries ${ }^{10}$. With regard to asymptomatic survival, then, in general, poorer outcomes are achieved after liver resections after the PVE compared to the primary resections without the PVE (ref. ${ }^{11}$ ). However, in some patients no increase of the liver parenchyma occurs, or in the worst case, a proliferation of metastatic processes occurs in the liver or the body ${ }^{12,13}$. Therefore, the therapeutic aim is to achieve growth of the FRLV as soon as possible in a short period of time after the PVE and to avoid tumor growth in the liver or the body. Support mechanisms can help to stimulate proliferation of the liver tissue either by direct cell division or through paracrine effects on the liver parenchyma.

Stem cells are represented in the adult population in all tissues and for clinical use these include blood, fat and bone tissue because their main sources are easily available. Circulating HSCs, whose source is bone marrow, are characterized by surface markers CD 34+ and CD 133+, and glycoproteins essential for mutual adhesion of cells (cell-cell adhesion interaction). It is known that these cells are heavily washed out into the peripheral blood after major surgeries. Based on the current experience in regeneration of liver parenchyma it is known that autologous HSC CD34+ and CD133+ are capable of differentiation into hepatocytes, Kupffer cells, cholangiocytes and endothelial cells $^{14,15}$. The role of HSC in the regeneration of the liver parenchyma was demonstrated in experimental studies ${ }^{16}$ in an animal model of acute liver failure. Nevertheless, there is a lack of clinical studies investigating the administration of stem cells in combination with PVE in association with liver regeneration. Most are rather isolated studies $^{17,18}$.

One major problem of the administration of HSC may be stimulation of metastases not only in the liver but possibly as micrometastases in the body. Tumor tissue contains a large amount of stimulating substances (growth factors, matrix-metallopropteinases, cytokines) which may cause the migration of HSC into the tumour tissue where they may further stimulate the growth and spread of the tumour. The mechanism of interaction of HSC with the tumor cells is not yet known exactly ${ }^{19}$. Probably a stimula- 
tion of neoangiogenesis with immunosuppression ${ }^{20}$ and inhibition of apoptosis occurs ${ }^{21}$. A likely mechanism which supports the growth of a metastasis in the liver includes differentiation of mesenchymal cells at the tumor site, in the so called carcinoma associated fibroblast (CAF) like cells and these cells then promote tumor growth ${ }^{22}$. Tumor growth can be facilitated by regenerative mechanisms of their own liver tissue after the PVE, when a variety of growth factors, metalloproteinases and cytokines is formed in the liver which can stimulate and facilitate proliferation of the tumour tissue $\mathrm{e}^{23,24}$ at the same time.

There are a lot of open issues related to tumor proliferation within the regenerative processes in the liver parenchyma. Undoubtedly, neo- and adjutant oncological therapy in the context of the PVE with or without administration of HSC plays its role. Current knowledge indicates that systemic chemotherapy based on fluorouracil with or without oxaliplatin, irinotecan, bavacizumab administered concurrently with the PVE is not, in most cases an obstacle for hypertrophy of the non-embolized lobe, and it is, to some extent, a fuse before proliferation of the liver tumour or systemic micrometastases in the interval needed to increase the non-embolized lobe following the PVE. Other studies should determine the effects of concomitant administration of HSC following the PVE and chemotherapy and biological therapy regarding regeneration of the liver parenchyma.

A metastatic focus in the pulmonary parenchyma occurred in our patient approximately 8 months following the PVE with administration of HSC and subsequent radical surgery. A question can be raised of course whether this development was a normal cancerogenesis process as it is generally known in many patients treated for CLM, or whether the development of pulmonary metastasis was stimulated by the mechanisms of regeneration/oncogenesis following the PVE with application of HSC. Currently, our study is ongoing on a larger number of patients and it should provide valid results in the near future.

\section{CONCLUSION}

The patient is presently 18 months after starting complex oncosurgical treatment without any signs of malignancy and in the very good condition. We believe, that he had no chance for long-term survival without the presented complex oncosurgical approach. Therefore all oncosurgical means to extend the quality of life in patients with CLM are worth trying.

\section{ACKNOWLEDGEMENTS}

This study was supported by the IGA MZ NS 9727 and IGA MZ NS 10240 grants.

\section{REFERENCES}

1. Clavien PA. Malignant liver tumors. Third Edition, Chichester, WileyBlackwell Publ; 2010.

2. Treska V, Skalicky T, Sutnar A, Liska V. Surgical treatment of colorectal liver metastases. Rozhl Chir 2009;88:69-75.

3. Carpizo RD, D'Angelica M. Liver resection for metastatic colorectal cancer in the presence of extrahepatic disease. Ann Surg Oncol 2009;16:2411-21.

4. Skalicky T, Treska V, Spidlen V, Vodicka J, Sutnar A, Liska V, Klecka J, Simanek V, Safranek J, Fichtl J, Polak M. Surgical treatment of liver and pulmonary colorectal metastases. Rozhl.Chir 2010:89:253-5.

5. Yokoyama Y, Nagino M, Nimura Y. Mechanisms of hepatic regeneration following portal vein embolization and partial hepatectomy: $\mathrm{A}$ review. World J Surg 2007;31:367-74.

6. Liu H, Shaihong Z. Present status and future perspectives of preoperative portal vein embolization. AJS 2009; 197: 686-90.

7. Hayashi S, Baba Y, Ueno K. Acceleration of primary liver tumor growth rate in embolized hepatic lobe after portal vein embolization. Acta Radiol 2007;48:721-7.

8. Belghiti J, Benhaim L. Portal vein occlusion prior to extensit resection in colorectal liver metastasis: A necessity rather than an option. Ann Surg Oncol 2009;16:1098-9.

9. Jaeck D, Bachellier P, Nakano H. One or two-stage hepatectomy combined with portal vein embolization for initially nonresectable colorectal liver metastases. Am J Surg 2003;185:221-9.

10. Wicherts DA, de Haas RJ, Andreani P, Sotirov D, Salloum C, Castaing D, Adam R, Azoulay D. Impact of portal vein embolization on longterm survival of patients with primarily unresectable colorectal liver metastases. Br J Surg 2010;97:240-50.

11. Gulik T, Esschert JW, Graaf W, Lienden KP, Busch ORC, Heger M, Delden OM, Laméris J, Gouma DJ. Controversies in the use of portal vein embolization. Dig Surg 2008;25:436-44.

12. Hayashi S, Baba Y, Ueno K. Acceleration of primary liver tumor growth rate in embolised hepatic lobe after portal vein embolisation. Acta Radiol 2007;48:721-7.

13. Pachos KA, Bird NC. Liver regeneration and its impact on posthepatectomy metastatic tumor recurrence. Anticancer Research 2010;30:2161-70.

14. Kallis YN, Alison MR, Forbes SJ. Bone marrow stem cells and liver disease. Gut 2007;56:716-24.

15. Fausto N, Campbell JS, Riehle KJ. Liver regeneration and repair: hepatocytes, progenitor cells, and stem cells. Hepatology 2004;39:147787.

16. Mark AL, Sun Z,Warren DS, Lonze BE, Knabel MK, Williams GM, Locke JE, Montgomery RA, Cameron AM. Stem cell mobilization is life saving in an animal model of acute liver failure. Ann Surg 2010;252:5916.

17. Esch JS II, Knoefel WT, Klein M, Ghodsizad A, Fuerst G, Poll LW, Piechaczek Ch, Burchardt ER, Feifel N, Stoldt V, Stockschlader M, Stoecklein N, Tustas RY, Eisenberger CF, Peiper M, Haussinger D, Hosch SB. Portal application of autologous CD 133+ bone marrow cells to the liver: A novel concept to support hepatic regeneration. Stem Cells 2005;23:463-70.

18. Kisseleva T, Gigante E, Brenner D. Recent advances in liver stem cell therapy. Curr Opin Gastroenterol 2010;26:395-402.

19. Ma S, Chan KW, Hu L, Lee TK, Wo JY, Ng IO. Identification and characterization of tumorigenic liver cancer stem/progenitor cells. Gastroenterology 2007;132:2542-56.

20. Peng N, Li L, Cai X, Tan S, Wu T. Liver stem/progenitor cells in the canals of Hering: Cellular origin of hepatocellular carcinoma with bile duct tumor thrombi? Stem Cell Rev and Rep 2010;6:579-84.

21. Harun N, Nikfarjam M, Muralidharan V, Christophi Ch. Liver regeneration stimulates tumor metastases. J Surg Res 2007;138:284-90.

22. Mishra PJ, Mishra PJ, Humeniuk R, Medina DJ, Alexe G, Mesirov JP, Ganesan S, Glod JW Banerjee D. Carcinoma- associated fibroblastlike differentiation of human mesenchymal stem cells. Cancer Res 2008;68:4331-9.

23. Cantz T, Manns MP, Ott M. Stem cells in liver regeneration and therapy. Cell Tissue Res 2008;331:271-82.

24. Liska V., Slowik P, Eggenhofer E., Treska V., Renner P., Mirka H., Kobr J., Sykora R., Schlitt H.J., Holubec L., Chlumska A., Skalicky T., Matejovic M., Dahlke M.H. Intraportal injection of porcine multipotent mesenchymal stromal cells augments liver regeneration after portal vein embolization. In vivo 2009; 23,229-36. 